\title{
EL QUEHACER PEDAGÓGICO, UNA REFLEXIÓN PARA EL EJERCICIO DE LA DOCENCIA CON LOS EMBERAS KATIOS
} PEDAGOGICAL ACTIVITY, A REFLECTIO FOR THE EXCERCISE OF TEACHING WITH THE EMBERAS KATIOS

\author{
María Helena Ramírez Cabanzo' \\ Fundación Universitaria del \\ Área Andina
}

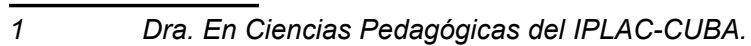
Magister en Educación, Universidad Santo Tomás, Especialista en Docencia Universitaria y Didáctica de la Enseñanza de la Historia, Licenciada en Ciencias de la Educación. mramirez75@areandina.edu.co. Docente de la Facultad de Educación de la Fundación Universitaria del Área Andina. Bogotá - Colombia. Líder del grupo de investigación Kompetenz, clasificado por Colciencias. https://orcid.org/00000002-1183-9069// https://scienti.minciencias.gov.co/cvlac/ visualizador/generarCurriculoCv.do?cod $r h=0000001463$ ${ }^{*} E l$ artículo corresponde a las conclusiones del proyecto financiado por la Dirección Nacional de Investigación de la Fundación Universitaria del Área Andina. Bogotá - Colombia. 2019. Código CVF2019-KO-B29.

Proyecto Transformación del quehacer pedagógico mediado por las geografías de la infancia: la experiencia de estudiantes y egresados Embera katíos de la Licenciatura en Pedagogía Infantil del Área Andina. Orientado por la investigadora María Helena Ramírez Cabanzo y Lorena Cardona Alarcón. Con la población de la Comunidad Embera Katíos de Frontino Antioquía, estudiantes y egresados de la Licenciatura en Pedagogía Infantil.

\section{RESUMEN}

Este artículo reflexiona sobre las prácticas pedagógicas con los Embera Katíos; tiene por objetivo general analizar procesos de transformación del quehacer pedagógico mediado por las geografías de la infancia: la experiencia de estudiantes y egresados Embera katíos de la Licenciatura en Pedagogía Infantil de la Fundación Universitaria del Área Andina. Inicia por el reconocimiento de escenarios de enseñanza aprendizaje mediados por la interculturalidad; donde se socializa diversos elementos que los estudiantes de 
la Licenciatura que se desempeñan como docentes, en instituciones educativas ubicadas en los resguardos del territorio donde se ubica la comunidad Embera Katíos; de igual manera se mencionan acciones del quehacer pedagógico intercultural para caracterizar la formación teórica práctica de docentes indígenas mediador por la virtualidad.

Cabe anotar que el ejercicio de indagación está en la fase III, y permite reconocer que la educación va más allá de la mera trasmisión de conocimientos en contextos de pueblos indígenas, se convierte en un escenario de formación centrado en el reconocimiento de valores y principios centrados en la cosmovisión de la comunidad indígena, conduce a mirarse así mismo, para luego reconocerse como maestros, mirarse en retrospectiva para identificar saberes que circulan en los pueblos originarios, para este caso, desde la propuesta institucional mediada por la virtualidad; en cuanto al diseño metodológico, se expondrán resultados que llama la atención del quehacer pedagógico de profesionales docentes desde una mirada interculturalidad, producto de las narraciones autobiográficas y exposiciones de los estudiantes como parte del trabajo final para graduarse.

Finalmente, el artículo señala conclusiones encontradas sobre aspectos generales de la pedagogía como docencia, didáctica, enseñanza- aprendizaje, así como el proceso de formación desde un tono reflexivo y consciente de las prácticas pedagógicas en contextos geográficos que merecen ser reconocidos. Así, el propósito general es aportar a la reflexión sobre formación docente y del quehacer pedagógico en contextos de comunidades indígenas.

\section{PALABRAS CLAVE.}

Quehacer pedagógico, Práctica Docente,
Enseñanza-Aprendizaje, formación.

\section{ABSTRACT \\ THE PEDAGOGICAL ACTIVITY, A REFLECTION FOR THE EXERCISE OF TEACHING WITH THE KATIOS EMBERAS}

\section{SUMMARY}

This text approaches to reflect on the pedagogical practices with the Embera Katíos; Its general objective is to analyze processes of transformation of the pedagogical task mediated by the geographies of childhood: the experience of Embera Katíos students and graduates of the Bachelor's Degree in Children's Pedagogy of the University Foundation of the Andean Area. It begins with the recognition of teachinglearning scenarios mediated by interculturality; where diverse elements are socialized that the students of the Degree who work as teachers, in educational institutions located in the resguardos of the territory where the Embera Katíos community is located; likewise, actions of intercultural pedagogical tasks are mentioned to characterize the theoretical and practical training of indigenous teacher's mediator by virtuality.

It should be noted that the exercise of inquiry is in phase III, and allows us to recognize that education goes beyond the mere transmission of knowledge in contexts of indigenous peoples, it becomes a training scenario focused on the recognition of values and centered principles in the worldview of the indigenous community, it leads to look at oneself, to later recognize themselves as teachers, to look in retrospect to identify knowledge that circulates in the native peoples, in this case, from the institutional proposal mediated by virtuality; Regarding the methodological design, results will be presented that draws the attention of the pedagogical work of teaching professionals from an intercultural perspective, product of the autobiographical narratives and expositions of the students as 
part of the final work to graduate.

Finally, the article draws conclusions found on general aspects of pedagogy such as teaching, didactics, teaching-learning, as well as the training process from a reflective and conscious tone of pedagogical practices in geographical contexts that deserve to be recognized. Thus, the general purpose is to contribute to the reflection on teacher training and pedagogical work in contexts of indigenous communities.

\section{KEYWORDS}

Pedagogical work, Teaching Practice, TeachingLearning, formation.

\section{CRUZANDO EL TERRITORIO,}

En Colombia, la oferta educativa comprende la complejidad de las regiones y se proyectan a una apertura académica según las necesidades de las comunidades; se expresa como nuevos logros ante la política pública para el país; en este marco, las instituciones de educación superior con programas modalidad virtual llegó al municipio de Frontino, departamento de Antioquía- Colombia y ofertó el programa de Licenciatura en Pedagogía Infantil, con la comunidad indígena Embera Katíos, quienes ingresaron a estudiar en medio de múltiples vicisitudes propias de su contexto

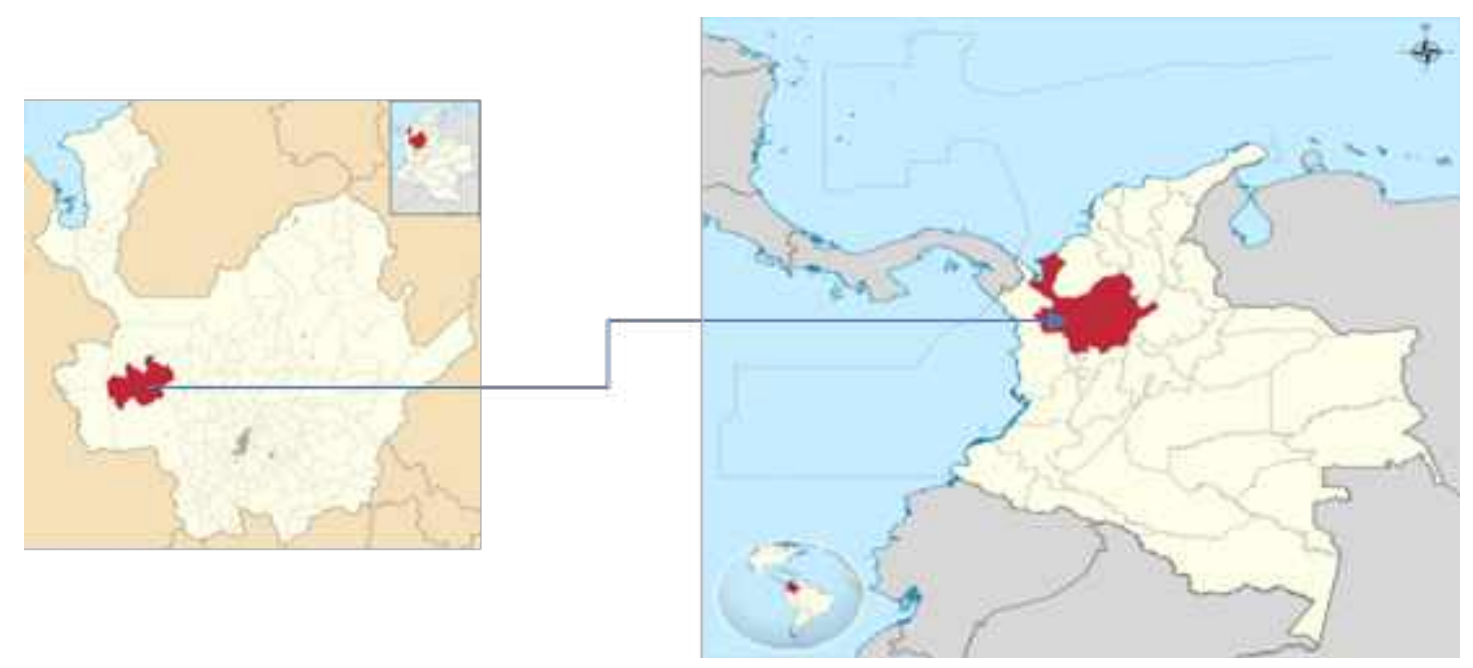

Imagen 1. Ubicación de Frontino en Antioquía y en Colombia

Tomado de: https://es.wikipedia.org/wiki/Frontino_(Antioquia)

La comunidad Embera Katíos se encuentran al noroccidente del departamento de Antioquía, próximos a Frontino y de los corregimientos de Uramita, Cañasgordas, Ubriaquí y Dabeiba, y otras veredas alejadas como Aguas Claras, Abrianqui, Julio Chiquito, Amparradó, Genaturado, Elano, Cañaverales, Murrí y Blanquita entre otros, rodeado por ríos que surcan el territorio alrededor de las proximidades del Parque Natural las Orquídeas. Lugar protegido de la acción del hombre, destinado a realizar actividades de carácter investigativo; cuenta con riqueza hídrica formada por los ríos Chaquenodá, Murrí y Sucio; en cuyas riberas se ubican los Embera Katíos; produciendo relaciones muy cercanas entre la comunidad y la naturaleza. (Ramírez, M. 2018). Otra forma de comprender a Colombia como un país multicultural, pluriétnico, con diversidad en las regiones y territorios que conducen a descubrir abismales diferencias sociales, económicas, culturales, educativas que merece ser comprendido desde la institución universitaria.

Así, la tarea se centra en reconocerlos como pueblos originarios con un cúmulo de saberes, su reconocimiento posibilita la construcción de otras epistemologías; de esta manera se configuran saberes de la comunidad, entre 
ellos, la mujer como portadora de conocimiento y formas de relación con los otros, con los mayores, los niños; las autoridades que participan del acto educativo y se esfuerzan por la preservación de la cultura, (Ramírez, M. 2018); todos, con la tarea de preservar la diversidad cultural que allí convergen y que aportan otra forma de comprender la realidad; para ello, diseñaron una ruta de aprendizaje de forma empírica desde el resguardar la lengua, la cultura y la relación con el territorio así como privilegiar saberes prácticos y formativos frente a las teóricos.

En este contexto, cabe indagar por la comprensión sobre el territorio y como despliega relaciones desde la pluralidad cultural para construir relaciones con otros saberes, desde el seno familiar en el tambo ${ }^{2}$, hasta el tambo donde funciona la escuela dentro de la comunidad. Así, el territorio transitado, el río al ser cruzado en lancha, nadando o en pequeñas embarcaciones que ellos elaboran proveen alimentos, animales, habitación y protección, en sus travesías, familias completas caminan para llegar a destino; llevan niños, jóvenes y los adultos con marcadas relaciones de protección y de apoyo:

"Los niños siempre están con sus padres, se les llevan para donde vayan, si es a estudiar, ellos saben que caminan todo el día, se hace el mercado, compran cositas que se necesitan" (palabras de Gloria Dominicó entrevista 11 de diciembre 2019, Facultad de Educación Fundación Universitaria del Área Andina)

"Yo siempre estoy con mis padres, conozco el camino, y el río, sé nadar desde chiquito, y el río es lo más divertido, juego, es todo" Palabras de niño de 7

$2 \quad$ Construcción cónica y circular, sin puertas, ni ventanas, con amplia visión al exterior, con escaleras para acceder a él, a una altura de 1 metro del suelo para protegerse de los peligros, con un fogón en el centro que permite el encuentro, vivienda propia de la comunidad Embera Katíos. años que siempre acompaña los padres y asiste a clase en Frontino.

El territorio para ellos, es amplio, repositorio de relaciones sociales que han generado un cordón umbilical con el espacio, Arfuch comenta, son una trama de interacciones a partir de la relación con el agua, el campo, los cultivos; son formas de ser, sentir y estar; esto posibilita estar cohesionados para compartir identidad desde la tradición oral (2007).

Al identificar los territorios como escenario de aprendizaje, se convierte en alternativas pedagógicas que se suscitaban en la escuela de la comunidad, estas pertenecen al resguardo y pueden transformar prácticas convencionales de la escuela; exige comprender como la diversidad cultural aporta a pensar en estrategias didácticas contextualizadas que provocan la aprehensión de lo real, es decir, el contexto y las relaciones socioculturales explican lo que acontece y son recursos valiosos en la formación de los sujetos, se constituye en tejido de relaciones diversas que anuda un vínculo educativo que anida contenidos de la cultura, los sujetos de aprendizaje y de saber que apoyan la naturaleza de la identidad con el contexto desde los afectos, los sentidos, lo imaginado, lo pensado, lo hablado para proveer formas de asumir los espacios y los sujetos, (Cardona, \&. 2017)

En esta medida se transita el territorio, cada sujeto observa el espacio, lo recorre, lo vive, Maffesoli (1993), menciona como se convierte en un espacio, un locus centrado, que constituye un sujeto sobre el espacio y que da sentido a su existencia socio histórica, esta idea resaltar el papel de las relaciones que entretejen sujetos convencidos de su papel histórico en relación con el territorio que recoge como tinaja los pasos de los mayores, los saberes de los Jaibaná, las huellas de los muertos, el retorno de la magia, de la relación hombre naturaleza en el contexto 
donde habita la comunidad Embera.

Cobra relevancia la afirmación de Maffesoli al comprender dos aspectos con los Embera Katíos, uno es el espacio, el territorio; otro, las relaciones que se tejen en ese espacio a partir de la experiencia de transitarlo con las familias enteras, para poder entender el territorio desde las transformaciones de los sujetos en la medida que se forman como docentes y desde el espectro de nuevo conocimiento; esto implica repensarlo por la responsabilidad que asume con el espacio, los sujetos, la comunidad, la institución (Ramírez, M. 2018). Cabe anotar que el concepto responde a espacio geográfico para consolidar el proyecto de la comunidad, que articula vidas personales y vidas colectivas, con entornos y situaciones que coexisten para constituir el proyecto de comunalidad, palabras de Medina (2011); es decir, una relación umbilical con sujetos, relaciones y espacio dentro del contexto histórico.

Desde esta perspectiva, el territorio oferta otras realidades, a partir de las ocupaciones que se hacen en el transitar, aportando experiencias a cada uno de los acompañantes; construye una relación dialéctica en torno a los conocimientos, los contextos y los sujetos. Para ello, las ideas expuestas por Pozo (2013), son pertinentes cuando expone que la educación reconoce las necesidades del contexto y centra acciones para atender a las comunidades en especial aquellas centradas en infancia; en este caso con población indígena; es decir, la universidad llega para reconocer saberes y fortalecer el concepto de educación propia, de la comunidad; como propuesta pedagógica determinada por las características sociales, culturales de la comunidad.

Razón de ello, los estudiantes de la Licenciatura exponen las propuestas pedagógicas a partir de lo cotidiano, lo que se transmite de generación en generación como una forma de recrear saberes heredados, transmitir a menores $y$ otros, que en conjunto reafirma la identidad cultural y la cosmovisión ancestral de toda la comunidad, es crear a partir de la experiencia que desencadena procesos de armonía con los todos los espacios que habitan y facilita la construcción de conocimiento como un elemento que caracteriza el componente pedagógico y explicar el quehacer de los docentes indígenas; tal como afirma (Tique y otros, 2017), los docentes se hacen en el ensayo y el ensayarse, para poder disponer de aprendizajes adquiridos en los diversos contextos como acontece con los Emberas Katíos, donde el contexto oferta posibilidades de cambio social y forma sujetos que transforma y a la vez crea nuevos escenarios en sus entornos

\section{EL QUEHACER PEDAGÓGICO}

Esta reflexión retoma el proceso educativo, para comprender el quehacer pedagógico; afirma el profesor Leal, no basta una buena formación en el saber de una disciplina, de una profesión o de un campo de investigación, sino que es necesario saber interactuar, generando situaciones de aprendizaje y retoma la formación de formadores combinando la formación pedagógica- y la acción comunicativa para acercar la realidad del contexto a quienes están aprendiendo.

La pedagogía vista como un campo reflexivo, interdisciplinar y crítico facilita el proceso educativo, lo hace significativo para quien aprende, permite al docente ser un guía en dicho proceso, un mediador cultural, encaminar valores y otros requerimientos en una formación integral, por lo cual requiere un engranaje entre lo teórico y lo práctico, deben estar presente en la formación del profesorado, afirma Leal; sin el quehacer pedagógico, el proceso educativo perdería su sentido y se limitaría en sí a un proceso de mera trasmisión de conocimientos, la capacidad de interacción esencial que caracteriza la formación de docentes, da sentido al proceso educativo, en ello donde radica la 
urgencia de generar programas de formación del profesorado fortaleciendo componente pedagógico esencial para todo contexto, (Tique y otros, 2017)

La superación de muchas de las dificultades que se presentan en el proceso educativo podrían ser revisadas a partir de la constante actualización y formación de los docentes, tanto en el componente disciplinar como en el quehacer pedagógico; esta situación es esencial para la formación básica, requiere actualización permanente por los requerimientos de una realidad cambiante. Y sigue el Dr. Zambrano Leal, "Si bien los profesores, en general, cuentan con una buena formación profesional o disciplinaria $y$, por lo tanto, manejan con propiedad $y$ responsabilidad el saber de su campo de acción, no siempre se han detenido a pensar en las posibilidades de mejorar su desempeño como docentes, es decir, no siempre saben enseñar lo que saben". Y continua diciendo, "Generalmente se recurre a la clase magistral o expositiva que, con frecuencia, ha contribuido a mantener una actitud marcadamente receptiva o pasiva por parte de los estudiantes; para cambiar esa actitud se ha venido consolidando una nueva tendencia que busca fomentar la participación activa de los estudiantes en su proceso de formación, recurriendo a otras estrategias metodológicas, lo cual ha puesto de relieve la necesidad de re conceptualizar y transformar el ejercicio de la práctica educativa del nivel superior, es decir, la necesidad de enfrentar el problema de la formación y desarrollo de la capacidad pedagógica de los profesores universitarios". (Zambrano, 2017).

Desde este referente, el quehacer pedagógico con los Embera Katíos, fractura la teoría por las diferencias de contexto y merece un apartado para conocer el proceso educativo y las prácticas de enseñanza en un espacio de encuentro e interacción como son los resguardos indígenas dispuestos para que el aprendizaje que se espera sea apropiado e integrador según las particularidades de la comunidad.

Desde la formación y encuentro que se tenían con los estudiantes de licenciatura y docentes en ejercicio se veía como una oportunidad para que desde las descripciones y las narraciones que explicaban el hacer en la comunidad se encontró de gran relevancia los significados que subyacen alrededor de la práctica y del quehacer en las acciones que desarrollan con los sujetos que asisten a la escuela, que producen saber pedagógico desde el hacer en contexto y la experiencia que a diario tienen para resguardar la cultura.

Es un saber pedagógico construido en la interacción con el contexto, da valor al propósito de construir como pensar otras acciones y reconstruir el pensar desde prácticas de enseñanza, afirma Cárdenas (2009), la práctica merece reflexión para pensar en sujetos, contextos y aprendizajes. Estas son posibilidades para mejorar el quehacer pedagógico del docente en contexto y optar por decisiones transformadoras de comunidad.

Así el docente de la comunidad Embera Katíos tiene la responsabilidad de fomentar los principios y valores de la comunidad como el circulo de la palabra para poder prepararse en las tareas de la comunidad;

"es necesario formar generaciones y que vayan hasta la universidad para que luego sean quienes ayuden a cuidar la cultura de todos". (Palabras de Pascual Bailarín, docente. Centro educativo Rural indigenista Julio Grandemos en el Corregimiento de Murrí,)

Por otro lado, hay que ofrecer todos los aprendizajes que se puedan de los antepasados, así se puede construir ambientes para construir el pensamiento de los mayores y de los muertos. 
Es una tarea del profesor de la escuela, pero también de todos los que pertenecemos a la comunidad, para desarrollar habilidades y aplicarlas en cada tambo y en cada familia; solo así se puede fortalecer el grupo de trabajo y aplicar en el contexto donde interactúa afirma el Profesor Pascual Bailarín.

Desde esta perspectiva se piensa en el sujeto de la comunidad en sujeto pedagógico, es un docente que transita entre la formación dada por la cultura, la formación institucional, la cualificación para convertir al docente en un apoyo para la cultural, para la pedagogía y para la educación como sistema en general. Es decir que el docente que se forma, pone en juego habilidades e imaginación, pasión, experiencias, que se hacen práctica con el intercambio de saberes para transformar contextos, otra forma de producir conocimiento, reconocer las tramas de la cultura para potenciar el acontecimiento educativo de la formación humana, afirma Ramírez-Cabanzo (2018)

El proceso de formación que tienen los docentes de la comunidad Embera Katíos, conduce a reflexiones sobre el quehacer pedagógico en la interacción con la comunidad, con los otros, en medio de los recorridos por el territorio, por las tradiciones, por las instituciones, que desencadena reflexiones sobre su hacer en un momento histórico de frente a realidades, en palabras de Freire, otorga emociones y sensibilidades para comprender a los sujetos y la forma de asumir compromiso con el contexto.

\section{EL QUEHACER PEDAGÓGICO CON LOS EMBERA KATÍOS}

Se retoma el concepto de educación, como espacio vivo que se construye como amalgama entre sujetos y la reproducción cultural; es permanente y tiene la tarea de socializar múltiples valores culturales; para los Embera
Katíos, es una forma de reconocimiento al conocimiento ancestral; retoma el tema de la tierra, desde la importancia de contener la historia como pueblo ancestral, en ella se concentra la defensa de la tierra para los hijos y el poder de la lucha por los otros, aquellos que están y vienen en camino; de esta manera, da fuerza a los saberes y sumados, amplían la cosmovisión de la comunidad y la diferencia de otras; tienen definida la valoración de la lengua originaria para proveer de forma colectiva la construcción del conocimiento desde adentro, en forma de círculo-espiral;

"así colocan en el centro a los mayores y sus orígenes y en la parte externa a los otros, mujeres, jóvenes y por ultimo a los niños, como una forma de exponer un proceso pedagógico que está en permanente construcción y deja entrever la autonomía de un currículo flexible, por tiempos, calendarios, formas de acceder a lo programado según el contexto y el reconocimiento de la participación comunitaria. (Palabras de Valentín Sinigui Bailarín, Líder de la comunidad).

A partir de los Jaibanas, los saberes se transmiten de generación en generación; se adjudica la tarea de enseñar al docente de la comunidad que se está formando para la tarea de enseñar, no como el lugar donde se aprende sino se comparte los saberes con la comunidad, hay madres que llegan y comparten saberes de forma abierta, todo el tiempo está en el tambo que es la escuela, acompaña los hijos;

"son sabedores y con los docentes son hacedores en el contexto; ellos desprenden $y$ vuelven aprender como parte del compromiso con la comunidad, no es curricularizado sino espontaneo". (Palabras de Pascual Bailarín, docente. Centro educativo Rural indigenista Julio Grandemos en el Corregimiento de Murrí,)

Esta forma de escuela dentro de la comunidad devela prácticas pedagógicas próximas al liderazgo de cada docente y constructor de 
un tejido para que la práctica prospere; cabe exponer la idea de Kemmis (2010), al expresar que las prácticas no son intencionadas sino producto de las circunstancias que rodean a cada participante, se deben a los contextos y las formas de interacción entre los sujetos. Desde esta perspectiva los docentes Embera Katíos, se convierten en sujetos activos en la comunidad que posibilitan transformaciones para todos. Así entretejen prácticas que centran la idea de la reflexión del quehacer docente y saberes adquiridos en el proceso de formación $\mathrm{y}$ al interior de la comunidad.

A ello suma la comprensión de una escuela en el Tambo, de puertas abiertas que permite reflexiones en relación directa con lo que acontece en la comunidad y los rituales que en ellas se suscitan como una forma de interacción educativa que articula conocimiento práctico con la planificación del proceso de enseñanza y otros elementos que el docente implemente de los libres, como evaluación, proceso de enseñanza, intención docente, que siempre son interdependientes, otra mirada para formar la práctica, que reconoce como parte del currículo intenciones, creencias, mitos, actividades como la cestería, la elaboración de chaquiras, la pintura facial, bailar y cantar como preparación para los ritos.

Cabe mencionar que gran parte de la formación inicia desde el vientre con el Nẽpoa, como ceremonia en la niñez, utilizando la naturaleza, acompañado por la familia, para otorgar habilidades al niño, o niña y prepararlo para vivir en la comunidad, protegerlos de enfermedades. Desde esta práctica y otras que se hacen al interior del Tambo; la escuela fortalece la construcción colectiva de saberes que despliega relaciones horizontales, define calendarios a partir del clima y la tierra para la cosecha, el tiempo y el espacio se definen por las fases lunares, ofrece espacios para la discusión, con voz para aportar a las soluciones de los problemas, los mayores y cabildantes van hasta las comunidades para hacerlos partícipes y reconocen sus aportes en discusiones del cabildo. Son insumos dentro de la educación propia para revitalizar principios y relaciones enmarcadas en valores, prácticas, saberes, que fortalecen la cosmovisión indígena, cumpliendo lo estipulado por el CRIC;

"las comunidades deben fortalecer la defensa de los procesos participativos como forma de exponer los saberes propios. (Palabras de Pascual Bailarín, docente. Centro educativo Rural indigenista Julio Grandemos en el Corregimiento de Murrí,)

Por otro lado, existe una preocupación por la recuperación de lenguas aborígenes a nivel latinoamericano; autoridades indígenas colombianas expresan interés por dar importancia a los conocimientos propios, así como la obligatoriedad del uso de la lengua, proponer la apropiación del espacio según lo planeado dentro de la comunidad,

"cambiamos de casa, ahora estamos lejos de Frontino, pero tenemos el río cerca y la huerta también" (palabras de Pascual Bailarín);

Cabe afirmar, la urgencia de materializar saberes en la práctica educativa de las comunidades a pesar del abismo entre el pensamiento ancestral y la política educativa que grita estar dentro de un modelo intercultural; al reforzar saberes como los mitos, las danzas, la historia, leyendas, pintura; los niños indígenas ya traen saberes que comparten con niños de otras comunidades, afirma Pascual Bailarín.

No se puede desconocer las tensiones que se producen entre los propuesto en la práctica pedagógica centrada en pensamiento ancestral y prácticas comunitarias y lo exigido en los procesos evaluativos por el Ministerio de Educación. Sin embargo, la posibilidad de 
proyectos educativos centrados en el rescate de los saberes de las comunidades otorgo la constitución de documentos que centren su interés en como la comunidad puede decidir sobre la política educativa de forma acertada y pertinente.

A través de las narraciones que hacen los estudiantes, docentes indígenas de su práctica pedagógica emerge el reconocimiento de un saber desde el contexto; retomando el concepto de didáctica sociocultural de Bombini (2006); que otorga contenidos culturales significativos para una comunidad según el contexto histórico de la misma. Es el camino para fortalecer identidades culturales propias de la experiencia de vivir, compartir, sentir y enseñar como la ruta de los docentes Embera Katíos. Es desde estas narraciones autobiográficas que surgen didácticas que aporta una comunicación desde el conocimiento tradicional y ancestral, que potencia modos de hacer desde lo que transcurre, como tarima para dar lugar a la identidad cultural de los sujetos que se encuentran en el aula, el Tambo; allí en medio de encuentro intercultural se fortalece lazos de comunidad y comunalidad, (Ramírez, M. 2018).

Para el mundo occidental es una propuesta novedosa que favorece una mirada de resistir todo lo occidentalizado; la concepción de Didáctica toma otro lugar y se concentra en otra forma de asumir lo que se enseña siempre desde la mirada del contexto, de cómo relacionar los problemas del contexto y la realidad de los sujetos para dar aplicabilidad en cualquier momento de la vida; a esto se añade la reflexión de los docentes de esas prácticas no solo pedagógicas sino socioculturales, es otra forma de apropiare de las problemáticas y proponer una "Intervención pedagógica desde la Investigación - Accción - Participación" el subrayado es mío; la razón, es la forma como se involucran con los problemas de la comunidad, entre todos diseñan estrategias mediadas y proponen soluciones para ser enseñadas a la comunidad y a los niños en la escuela, es sencillo y práctico, dice Pascual;

"se piensa como se hace y se enseña con todos, para que aprendan y puedan dar sentido a lo aprendido, es la construcción del saber y del aprendizaje en todo el resguardo que es aula y escuela; cuando estaba en la universidad aprendí como se llama lo que desde siempre hemos hecho". (Palabras de Pascual Bailarín, docente. Centro educativo Rural indigenista Julio Grandemos en el Corregimiento de Murrí,)

\section{EL RESCATE DEL SUJETO COMO DOCENTE TRANSFORMADOR,}

Este apartado, propone la transcendencia de los docentes de la comunidad Embera Katíos, como docente y sujeto que acompaña la comunidad, centra su tarea en la autodeterminación de la misma a partir del reconocimiento de recuperar la cultura y las tradiciones para preservarlas; Zemelman (2002) propone que el sujeto cumple la tarea de recuperarse como sujeto histórico, desde el acto de enfrentarse a la realidad, cuando hace reflexión epistémica siempre tiene una raíz histórica, es decir a partir de una necesidad histórica desde cosas elementales para comprender el momento histórico y ello significa preguntase por la persona que lee y transforma los contextos desde la realidad de vida y pensamiento, como se interpreta lo que acontece a partir de las condiciones sociopolíticas de contexto se erigen en la actualidad.

Frente a esto surgen desafíos para comprender el presente, de tiempo y espacio que es lo que acontece, allí en el tiempo determinado para comprender que la historia es un cumulo de presentes sucesivos, de construcciones cotidianas, observaciones permanentes, comprometidas, creencias, que tienen en su mano la tarea de hacer lo que otros no han 
hecho, que demuestra la ausencia del sujeto en el propia acción que asume acciones colectivas por encima de individualismo y ello abastece otra forma de pensar el presente como acto de pensamiento para entender el presente; razón de ello, los Embera Katíos, están en tarea de construir futuro como tarea propia del sujeto histórico, que allí concentra. La práctica es praxis de conciencia, es impactante, cambiante, facilita respuestas a diferentes situaciones $\mathrm{y}$, al mismo tiempo, brindar nuevas herramientas al docente, (Tique y otros, 2017), esta idea permite acercamiento al sentido su formación.

La práctica, entonces se aleja de la repetición, de la cotidianidad, implica preparación, pensar y pensarse en contexto, es compromiso - no solo un deseo de educar-; va más allá, es reconocerse como sujeto activo que implica a otros, a toda una comunidad; así la práctica implica conciencia y voluntad y se hace sujeto histórico, con capacidad de construcción, que anudan conocimiento, voluntad, emocionalidad y compromiso, con imaginación, intuición, que da sentido al rol en la comunidad y rompe con lo establecido.

El docente que se forma en la comunidad asume posturas como parte de la comunidad no desde su rol como docente; para los Emberas, son los sujetos que se atreven a construir conocimiento, es decir se autoconstruyen como sujetos dentro de los espacios existentes y los nuevos dentro de la realidad, una forma de dar lugar a la historia, a la realidad cotidiana, que se afrontan como hombres, que se reconocen por sus acciones, desde lo individual y lo colectivo, ello conduce a verlos como actores que transforman toda la comunidad, por las habilidades que posee y lo convierte en sujeto provocador de otras realidades para la comunidad. Es una forma de pensar la realidad del presente como un complejo entramado de manifestaciones donde transcurren sujetos, saberes, relaciones y espacios, que va desde la reconstrucción de la experiencia vivida en contexto que vive unos tiempos donde articula saberes de la comunidad y saberes, desde la formación como docente Licenciado de la Fundación Universitaria del Área Andina.

Es una forma de comprender la práctica pedagógica como la acción de reflexión de los sujetos, es decir se construye en un plano social y afectivo palabras de Carr (2006), las prácticas se adecuan a los contextos se modifican constantemente para los Embera Katíos, como medio para preservar la cultura y el territorio.

"pues en la interacción allá en el resguardo, da sentido a la práctica y la interpretación que damos a todo, a través del lenguaje, se apoya desde la relación con los todos los otros, con la cultura y los saberes previos, palabras de Pascual Bailarín; pero afirma ,que no es copiar todo de los libres sino dar cuerpo a los saberes ancestrales, porque nos debemos a los mayores y a los antepasados;

"Sin embargo, en la comunidad se preocupan por la preparación de los maestros, y los motivan ir a los microcentros, escuelas de formación para aprender más sobre el ejercicio de la docencia", palabras de Gloria Dominicó.

Ahora retomando a Carr, es necesario la suma de teoría y práctica para fortalecer la práctica educativa o de lo contrario carece de sentido. Por ello se organizó el microcentro, espacio donde se encuentran los maestros y maestras indígenas del municipio de Frontino para organizar, planear y evaluar sus planes de estudio, allí hablan de las dificultades en el ejercicio docente, así como lo que acontece en las instituciones que apoyan la educación indígena.

"En los últimos años, se amplió la participación de los integrantes y apoyan la junta del cabildo mayor, los cabildos locales, los representantes de los grupos conformados en las comunidades: mujeres, jóvenes, niños y niñas, la Organización 
Indígena de Antioquia regional, las hermanas misioneras de la Hermana Laura, quienes participan y apoyen la educación indígena", palabras de Gloria Dóminicó.

Siguiendo, a Kemmis, (2014); define la práctica como el conjunto de acciones que el docente desarrolla desde el contexto y permite apropiación de saberes y culturas para proveer las necesidades de la comunidad. Es reconocer un dialogo de saberes, para el caso de los Embera Katíos es dar valor a la cultura, validar su tiene su epistemología y merece dejarla florecer, estableciendo un equilibrio entre la diversidad de conocimientos, los diálogos entre sujetos y comunidades bajo condiciones justas que superen las relaciones coloniales de poder frente al conocimiento.

Estas ideas mencionadas, exponen ejes valiosos para el tratamiento del territorio y expresiones multiculturales que se inscriben en él, a partir de la subjetividad espacial, en los significados otorgados a este desde las prácticas que se desarrollan allí. Así, "el territorio no solo tiene una realidad material, sino una construcción sociocultural a través de procesos sociales que lo cargan con sentidos, significados y memoria en la vida práctica" Maffesoli.

En este contexto, el interés de investigación va acercándose a la conceptualización de territorio epistémico, con énfasis en un enfoque de diversidad cultural que propone otra reflexión. Así en cuanto a la formación docente, esta se mantiene en el umbral que ubica la atención en las relaciones entre los saberes de los maestros en formación a partir de la metáfora del territorio y su incidencia en las prácticas pedagógicas, y la apropiación del quehacer pedagógico en contextos particulares con los por parte de los docentes que se encuentran en contextos particulares como los Emberas Katíos, como componente fundamental para el proceso de aprendizaje; ser sujeto en contexto apropiado de la cultura que transita en la comunidad.

Hablar de la formación de sujetos vinculados al proceso educativo, propio del proceso de enseñanza-aprendizaje para los Emberas que se apoyan en la comunidad para que el aprendizaje de los otros se facilite; es promover espacio de reflexión, de conocimiento y reconocimiento del quehacer pedagógico que, valida el interés de todos para compartir experiencias pedagógicas, vivencias del docente y comprensiones de la comunidad.

Finalmente, los contextos se deben a sujetos que los transitan y proveen conocimiento $\mathrm{y}$ acción; los proyectos están direccionados para mantener la cultura de los pueblos indígenas, de forma pertinente y competente, que requiere procesos de formación de maestros desde el concepto de educación propia que reconozca la diferencia de cada contexto. EI $\mathrm{CRIC}^{3}$ desde 1971 dispuso las orientaciones de organizar un Programa de Educación Bilingüe Intercultural (PIBE). Devela la preocupación por la formación de docentes bilingües que eduquen desde la situación de los indígenas y en sus respectivas lenguas, (Vitonás, 2010).

\section{PARA TERMINAR,}

El contacto con la comunidad Embera Katío, ha promovido comprender la pedagogía como un espacio vivo, dinámico, donde muchas voces, intenciones, experiencias lo nutren; todo gira en la conservación de la cultural, preocupados por la no perdida de la lengua, por la formación de niños y niñas para mantener costumbres e identidad como pueblo originario.

El territorio transitado, la comunidad, el tambo, el río, los mayores, las abuelas con libros abiertos de aprendizaje permanente; son escenarios donde transita la cultura y los saberes para

$\overline{3}$ CRIC: Consejo Regional Indígena del Cauca. 
reafirmar prácticas culturales, sociales y pedagógicas.

Las cartografías individuales y colectivas develaron la trayectoria de un pueblo que se resiste; pero que la experiencia que acumula cada integrante de la comunidad, la experiencia vital que da sentido a su labor como docente.

Cabe mencionar a Zambrano, siempre hay que interrogar al docente para ponerlo en crisis y que pueda controvertir sobre la escuela, la enseñanza, los aprendizajes entre otros para colocar al docente Embera Katío como un mediador, artesano de la cultura y un puente de vida entre los mayores como libros abiertos y los niños y jóvenes integrantes de la comunidad.

Se reconoce esta indagación como una posibilidad de explorar saberes de las comunidades, en especial los Embera Katíos, es abrir una ruta hacia la denominada "Epistemología del Sur" donde las comunidades, las tradiciones, las danzas, el tejido, la pintura facial, sus ritos develan una diversidad cultural y en cada encuentro con ellos, las narraciones provocaron múltiples historias y refleja la pluralidad de la nación colombiana como cuerpo vivo que merece reconocimiento de su identidad cultural.

Esta indagación pone a circular otras formas de dignificar el ejercicio de la docencia desde las comunidades y los contextos, pues da ingredientes reales para hablar desde una perspectiva intercultural, ya que los conocimiento, niño, escuela y comunidad permite repensar las relaciones, las interacciones, los encuentros y desencuentros para ofertar otros actos del quehacer pedagógicos con los Embera Katíos.

Este ejercicio otorga tres elementos a otros investigadores; primero la interacción como forma de encuentro para resignificar el saber y el aprendizaje, así como a la academia para dar lugar prácticas investigativas en contextos socio diversos; segundo, el reconocimiento a la experiencia como el proceso de vivencia y emoción para conocer a esos otros que están al lado. Tercero, las narraciones de las experiencias, permite el encuentro con la comunidad, con el territorio y con los saberes ancestrales como una vía para que toda la nación la reconozca y se apropie, es la tarea del quehacer pedagógico con los Emberas Katíos desde una mirada intercultural, efectivamente una reflexión para el ejercicio de la docencia.

\section{REFERENCIAS BIBLIOGRÁFICAS}

1. Arfuch, L. (2007). El espacio biográfico. Buenos Aires. Fondo de Cultura Económica.

2. Bajtín, M. (1997). Hacia una filosofía del acto ético. De los borradores y otros escritos. Barcelona: Anthropos.

3. Bombini, G. (2006). Prácticas de lectura. Una perspectiva sociocultural. En O. Vallejos, G. Bombini, L. Zimmermann, A. Falchini, F. Mónaco, D. Riestra y otros, Lengua y Literatura. Prácticas de enseñanza: perspectivas y propuestas (pp. 27-43). Argentina. Santa Fe: Universidad Nacional del Litoral.

4. Cárdenas, P.A. (2009). Literatura, pedagogía y formación en valores. Enunciación. Revista Enunciación. Literatura en el contexto Educativo. 14, Na. 2, pp. 5-20. https://doi. org/10.14483/22486798.3057

5. Cardona, L. Ramírez, M. Vergara M. y Gutiérrez, A. (2017). Geografías del cuidado y la crianza desde la experiencia educativa en Comunidades originarias de América Latina. Fundación Universitaria del Área Andina. Facultad de Educación. Dirección Nacional de Investigación. Bogotá. 
6. Carr, W. (2006). Education without Theory. British Journal of Educational Studies, $N^{a}$ 54: 2; $136-159$.

7. CRIC. Consejo Regional Indígena de Colombia.

8. Del Pozo Serrano, F. J. (2013). Educación social para la salud: proyección, acción y profesionalización. Revista Médica Risaralda, 19(1), pp. 75-80

9. Kemmis, S. (2010). Research for Praxis: Knowing doing, Pedagogy, Culture \& Society. Vol. 18, Na . 1, pp. 9-27.

10. Maffesoli, M. (1993). The Social Imaginary. Current Sociology. Vol $411 \mathrm{~N}^{\mathrm{a}} .2 \mathrm{pp}$ $59-67$

11. Medina, P., López, S \& Ángeles, I. (2011). Comunidades-comunalidades. RevistaTRAMAS 34 p. 143-178. Tomado de: http://132.248.9.34/ hevila/TramasMexicoDF/2011/no\%2034/6.pdf

12. Pérez, A. (2002). El pensamiento del profesor. Vínculo entre la teoría y la práctica. Revista de Educación, 284, Madrid. pp. 199-221.

13. Ramirez-Cabanzo, A.(2018). Maestra Escuela: La investigación y el Maestro como Intelectual de la Pedagogía. Comp. Ramírez, Castiblanco, Velazquez. Red Chisua. Bogotá. SED.

14. Ramírez, M. Cardona, L. (2018). Los niños: sujetos de derecho en la comunidad Embera Katíos, de Frontino Antioquía, Colombia. En Diferentes geografias de la Infancia: Experiencias y vivencias investigativas en Latinoamérica. Colección Prácticas, Saberes y Conocimiento. Tomo 1. Fundación Universitaria del Área Andina, Bogotá Colombia.

15. Tique Calderón, A, Mozuca Ruiz, E y Alvarado González, D. (2017). Sistematización de la práctica en el programa de Licenciatura en Pedagogía Infantil. Fase 1. Bogotá:
AREANDINA. Fundación Universitaria del Área.

16. Vitonás, A. (2010). El PEBI, 39 años de construcción de una educación propia en Colombia. Revista Guatemalteca de Educación. Enero - Julio 2010.

17. Zambrano, A. (2017). Conferencia Magistral "Paz y Educación" Pre - Foro Educativos departamentales. Ministerio de Educación. Agosto 2017. Acceso en el link: https:// www.youtube.com/watch?v=liHWBARilk8

18. Zemelman, H. (2002). Necesidad de conciencia. Barcelona: Anthropos. 